\title{
La gestión de la calidad mediante métodos científicos
}

\section{Quality management using scientific methods}

\author{
GUTIÉRREZ-GARCÍA, Alfredo*† \\ ID $1^{\text {er }}$ Autor: Alfredo, Gutiérrez-García / ORC ID: 0000-0002-8197-8144
}

UNIDEG, Ingeniería Industrial, México. Ingeniería Industrial, de la Universidad Tecnológica Del Norte De Guanajuato, Blvd. Hidalgo 34, Universidad, 37800 Dolores Hidalgo Cuna de la Independencia Nacional, Gto

\section{Resumen}

Existen diversas herramientas para la gestión de la calidad de los procesos y servicios, las cuales tienen como fin común repercutir en la satisfacción del cliente, es este sentido, el procedimiento adecuado es identificar primeramente los críticos de la calidad (CTQ's), a fin de enfocar los esfuerzos en lo que represente más importante y aplicar QFD ., secundariamente a fin de obtener una lluvia de ideas, formular un diagrama de Forrester simulando la repercusión que las variables provocan en el bucle o critico de mayor importancia obtenido en el QFD para así comenzar con la investigación científica y utilizar los modelos estadísticos y de control que se ajusten a la raíz del problema encontrado.

QFD, Forrester, Diseño de experimentos, CHI cuadrado, TRIZ, Balanced ScoreCard

\begin{abstract}
There are several tools for managing the quality of processes and services, which have as their common purpose to have an impact on customer satisfaction. In this sense, the appropriate procedure is to first identify quality critics (CTQs), a In order to focus the efforts on what is most important and apply QFD., secondarily in order to brainstorm, formulate a Forrester diagram simulating the repercussion that the variables cause in the loop or critic of greater importance obtained in the QFD to begin with scientific research and use statistical and control models that fit the root of the problem encountered.
\end{abstract}

QFD, Forrester, Design of experiments, CHI square, TRIZ, Balanced ScoreCard

Citación: GUTIÉRREZ-GARCÍA, Alfredo. La gestión de la calidad mediante métodos científicos. Revista de Educación Técnica. 2019. 3-7: 19-26.

\footnotetext{
*Correspondencia al Autor (Correo Electrónico: alfredo.gutierrez@utng.edu.mx)

$\dagger$ Investigador contribuyendo como primer autor.
} 


\section{Introducción}

Hoy día, la gestión de la calidad es una fuente de investigación continua, pues, aunque se proponen una infinidad de soluciones a la mejora de los sistemas, estos generalmente no cumplen con su cometido sustancialmente., esto es derivado de la falta de investigación de carácter científica que se traduce en fracasos o costes altos para las empresas. Sin embargo, la investigación científica para aumentar la productividad no es precisamente una actividad catedrática., en la actualidad la tecnología ha diseñado aplicaciones que ejecutan los algoritmos extenuantes de muchas de las técnicas para la innovación, en este sentido, esto apoya a que cualquier persona con conocimientos medios de informática y con una breve capacitación, pueda simular, ejecutar e interpretar los modelos más complejos proponiendo una estrategia basada en análisis tanto matemáticos como estadísticos minimizando así el impacto negativo de las propuestas de mejora al mismo tiempo que baja los costos hundidos provocados por la falta de investigación guiada por intuiciones, falsas experiencias, históricos confusos e hipótesis sin fundamentos., en esta línea, en la presente investigación se dará una guía de 6 pasos para garantizar la confiabilidad de los resultados tomando como ejemplo una empresa de lácteos.

\section{Descripción de los métodos}

Los diseños de experimentos son modelos estadísticos que tienen como objetivo averiguar si unos determinados factores influyen en una variable de interés $y$, si existe influencia de algún factor cuantificarla, además de poner optimizar los resultados obteniendo la respuesta más viable. Por su parte QFD es una metodología creada por Shigeru Mizuno y Yoji Akao en Japón en los años 60 y tiene como propósito mejorar los procesos, productos o servicios de una compañía, producir un resultado más rápido que otros métodos y dar definición al proceso de diseño el cual se compone de diferentes partes como:

- La parte horizontal contiene la información del cliente.

- La parte vertical contiene información técnica que responde a información obtenida del cliente.
Por su parte el diagrama de Forrester es un diagrama de relaciones que permite analizar los vínculos de las causas y efectos de una situación problemática cuando se presentan de forma compleja y dar un panorama de cómo influyen las variables de entrada en las de salida, obteniendo así, las características que se deben optimizar en los modelos subsecuentes., en esta línea, la prueba de CHI cuadrado o Ji cuadrado (X2), es utilizada para el análisis de variables cualitativas. Su nombre lo toma de la distribución Chi cuadrado de la probabilidad, en la que se basa, fue desarrollada ya en 1900 por Pearson, y su utilidad es evaluar la independencia entre dos variables nominales $u$ ordinales, dando un método para verificar si las frecuencias observadas en cada categoría son compatibles con la independencia entre ambas variables, además de evaluar la probabilidad de existencia de discrepancia entre los datos y las frecuencias esperadas según la hipótesis nula. Finalmente, Balanced Scorecard (BSC), es un sistema de administración utilizado en la planeación estratégica, que puede ser utilizado en diferentes áreas, en este contexto, las cuatro perspectivas con las que cuenta la metodología tiene sus propios indicadores que sirven de parámetro para verificar que los objetivos planeados se estén cumpliendo para así llegar a las metas.

\section{Aplicación de la metodología a través del caso de estudio}

Primera fase: Para lograr la gestión de la calidad de manera eficiente, el presente caso de estudio se lleva a cabo en una empresa de elaboración de quesos, en este sentido se comienza con la implementación de la herramienta de QFD, la cual cuenta con una ponderación de (0 a 10), para a los QUES, (-1 0 y 1), para los COMOS, la relación de los QUE's con los COMOS (1 3 9) y la relación de los COMO's con los COMO's (- +), siendo menos y más importante respectivamente. 


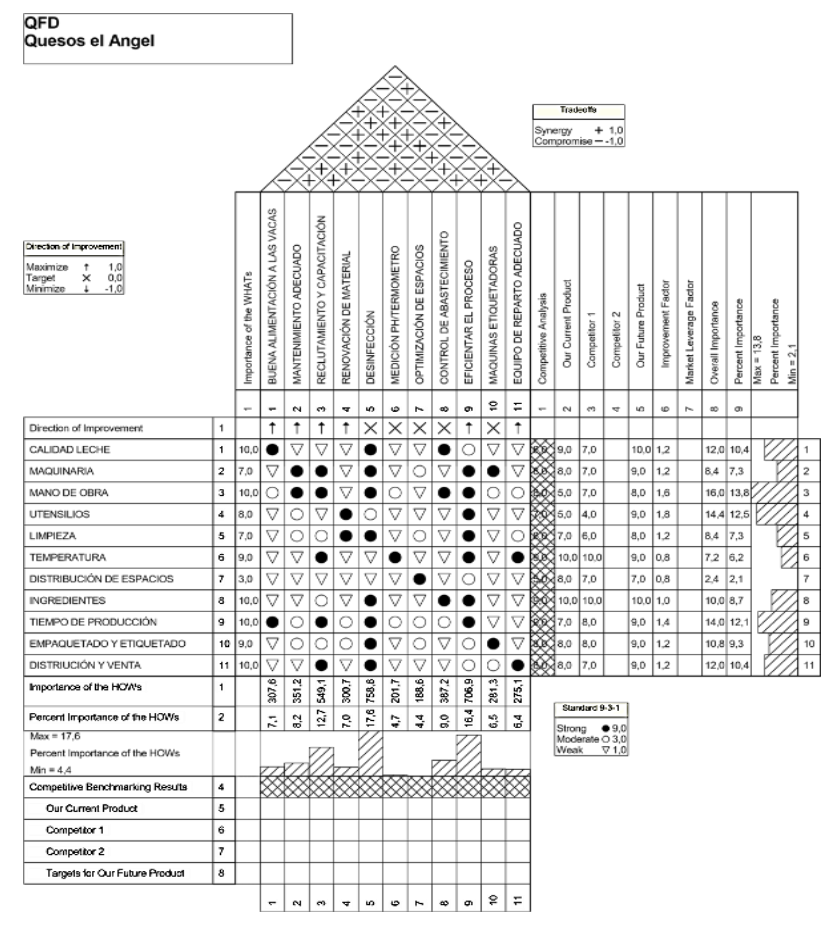

Figura 1 Casa de la calidad obtenida del QFD

Ahora bien, los resultados que se pueden observar en la casa de la calidad proporcionada por la aplicación de la metodología de QFD, es que el QUE y el COMO más importante en el proceso de elaboración de queso es la mano de obra y acciones salubres, es por ello que en lo siguiente se tomara de referencia este indicador y enfocar la investigación.

Segunda fase: Siguiendo con las técnicas de manera estructurada, ahora es necesario crear un diagrama de Forrester tomando como bucle el ya mencionado problema, mejorar el mano de obra, modelo en el cual se tomaron de referencia ponderaciones de $\left(\begin{array}{lll}0 & \text { a } & 1\end{array}\right)$ para medir el impacto y la importancia que cada una de las variables que influyen de manera sistemática.

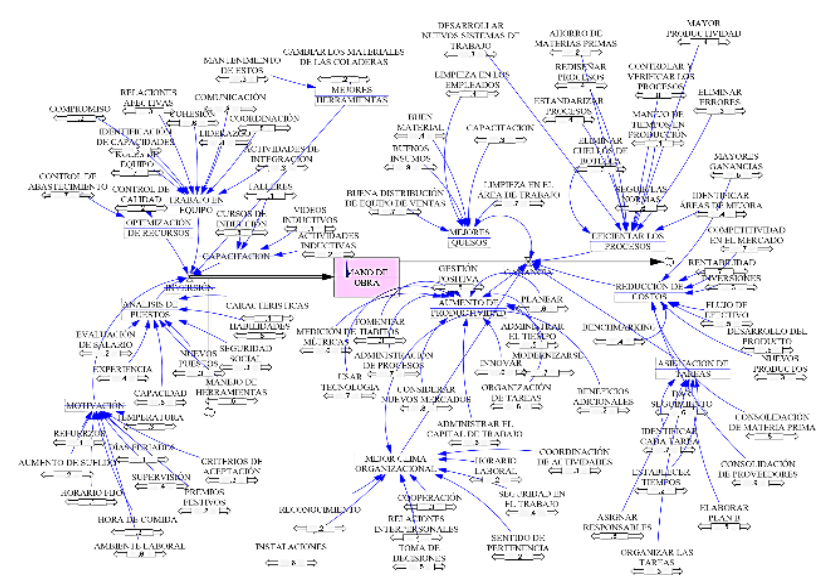

Figura 2 Simulación del diagrama de Forrester
Por consecuente, los datos simulados en el diagrama de Forrester indican que las variables de entrada que la empresa de lácteos tiene o que actualmente toma en cuenta para satisfacer este CTQ no son suficientes, pues el pulso del bucle se encuentra en un $20 \%$ por debajo del ideal para el modelo, es decir, como mínimo y para logar la satisfacción sin afectar las finanzas de la empresa, las entradas tiene $30 \%$ de falta de ajuste, por lo cual se están teniendo perdidas sustanciales tanto de tiempo como monetarias ya que estas no promueven el aumento de la producción., que, dicho de otro modo, el $50 \%$ es donde se debería de ubicar el pulso como mínimo para no tener pérdidas y de ahí en adelante, cualquier porcentaje es ganancia, eficiencia y eficacia de los sistemas., por lo tanto, la inversión en la mano de obra no proporciona las ganancias necesarias para mantener equilibrado el sistema en la empresa.

Tercera fase: Diseños de experimentos, este modelo estadístico tiene la finalidad de organizar las variables para identificar su repercusión en la mano de obra para obtener mejor productividad en la empresa de lácteos, en este sentido, esta es la variable de respuesta y el trabajo en equipo, la capacitación, la cultura y la motivación, serán las variables de influencia tomando de referencia los datos obtenidos de los dos semestres del 2017, los dos del 2018 y el primero de 2019.

\begin{tabular}{|l|l|l|r|l|l|}
\hline P eriodo & P ro ductividad & Trabajo en equipo & Capacitación & Cultura & Mo tivación \\
\hline ler semes tre 2017 & 1,02 & 0,37 & 8 & 0,52 & 0,2 \\
\hline 2do semes tre 2017 & 0,97 & 0,34 & 6 & 0,55 & 0,23 \\
\hline ler semes tre 2018 & 1,28 & 0,37 & 11 & 0,6 & 0,27 \\
\hline 2do semestre 2018 & 1,28 & 0,39 & 6 & 0,6 & 0,34 \\
\hline ler semes tre 2019 & 1,57 & 0,43 & 8 & 0,6 & 0,43 \\
\hline
\end{tabular}

Tabla 1 Ponderaciones a las variables independientes y la dependiente

\begin{tabular}{|c|c|c|c|c|c|c|c|c|c|c|c|}
\hline Productivids & \begin{tabular}{|l} 
Thabjo en \\
equipo
\end{tabular} & 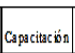 & Culurs & Motiactín & ortenesst & Oortencorite & it Bbques & Tpopt & AUSTIES1 $\mathrm{k}$ & RESI & COEF1 \\
\hline 102 & 0,37 & 8 & 0,52 & 0.2 & & 1 & 1 & 1 & 102 & $-2228-16$ & \begin{tabular}{l|l}
6.2824798 \\
\end{tabular} \\
\hline 097 & 0,34 & 6 & 0.55 & 0.23 & & 2 & 2 & 1 & 0.97 & & 0,0844023 \\
\hline$\underline{128}$ & 0,37 & ॥ & 0.6 & 0.27 & & 3 & 3 & 1 & $\lfloor 28$ & $-2,228-16$ & \begin{tabular}{|l|l|}
6,07800055 \\
\end{tabular} \\
\hline 128 & 0,39 & 6 & 0.8 & 0.34 & & 4 & 4 & 1 & $\lfloor 28$ & & of 0,0238449 \\
\hline (7) & $0: 43$ & 8 & 0.8 & 0,43 & & s. & s. & 1 & 1.57 & & 0.048869 \\
\hline
\end{tabular}

Tabla 2 Diseño de experimentos con superficie de respuesta 


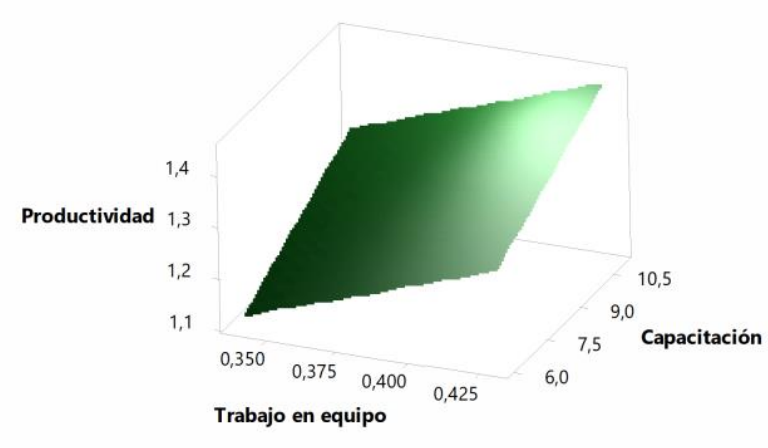

Figura 3 Superficie de respuesta Productividad, trabajo en equipo Capacitación

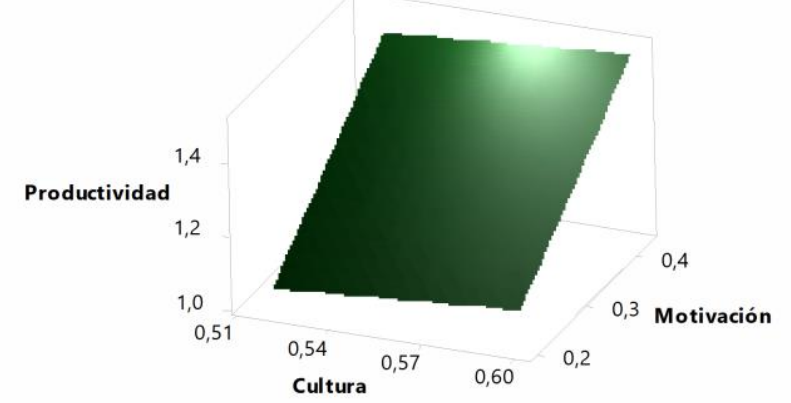

Figura 4 Superficie de respuesta Productividad, Cultura \& Motivación

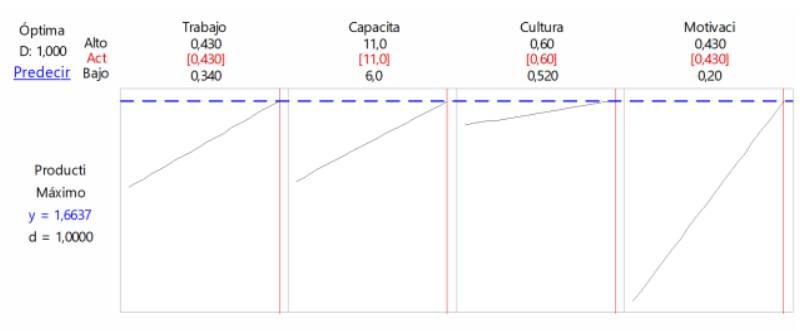

Figura 5 Optimización de la repuesta

Es cierto mencionar que el diseño de experimentos de superficie de respuesta, no propone ninguna opción optima al modelo, esto es debido a la complejidad de las variables, aun así, es justo mencionar que la confiabilidad del modelo es del $100 \%$, aun ajustado., por tal motivo, se ha buscado la optimización de modelo, para lo cual se tomaron como restricciones lo siguiente: para el trabajo en equipo, mantener el máximo valor del 0.43 , la capacitación entre los parámetros mínimo y máximo, es decir, entre 6 y 11 capacitaciones por semestre, los factores culturales en el máximo posible de 0.6 y la motivación del personal igualmente en el máximo de 0.43 .
Con lo cual es posible concluir que, la respuesta solución para mejorar la productividad en la empresa de lácteos maximizando las variables del sistema es: seguir con 11 capacitaciones semestrales, con lo cual la motivación se mantendrá en su máximo posible, además del trabajo en equipo y la cultura empresarial, en este sentido, esto proporcionará un aumento en la productividad posicionando se en 1.6637 como se puede observar en la figura número 5 .

Cuarta fase: Chi cuadrado, este análisis tiene la finalidad de identificar si las variables analizadas en la presente investigación, son o no significativas y si están relacionadas entre sí, en este sentido, se han tomado de referencia las variables de productividad, trabajo en equipo, capacitación, cultura, motivación y costos obteniendo una matriz de $5 \times 6$.

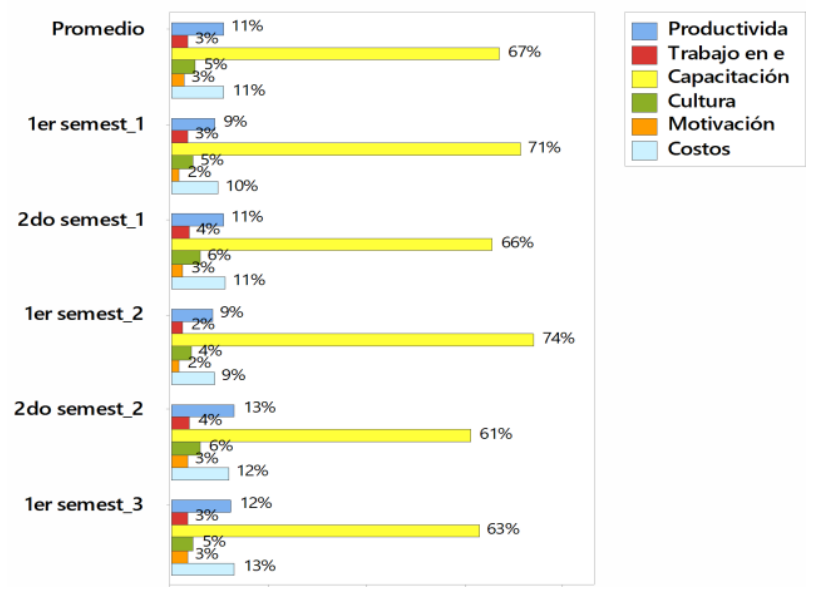

Figura 6 Resultados de prueba $\mathrm{X}^{2}$ datos en porcentajes

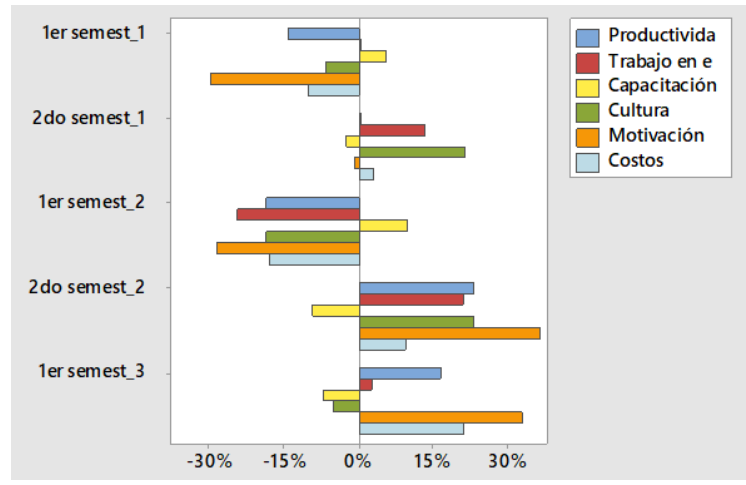

Figura 7 Porcentaje de ocurrencia de prueba X2 
Con la prueba de hipótesis chi cuadrado es posible identificar que existen diferencias significativas en las variables estudiadas, pues la productividad no ha sido equiparable con la cantidad de capacitaciones que se han tenido, el primer semestre del 2017 existieron más capacitaciones y la productividad majo drásticamente, al igual que en el segundo semestre del 2018, donde fueron menos capacitaciones y más productividad, es por ello que se debe de poner énfasis en el tiempo de capacitación que se imparta, ya que estadísticamente, estas no han sido productivas todas propiamente, en este sentido, esto ocurre con bastante frecuencia en las demás variables investigadas aceptando la hipótesis alternativa con $95 \%$ de confiabilidad del modelo, oh dicho de otro modo, hay que prestar atención los tipos de capacitaciones que se han impartido,. por consiguiente, las capacitaciones de este periodo corresponden a una interna y una más externa., dos evaluaciones formativas $y$ de pos evaluación, representadas en los anexos.

Quinta fase: Identificar mediante la técnica TRIZ, los principios de inventiva que sean aplicables de los 40 totales y que son marcados por los 39 parámetros de Altshuller mediante la matriz de contradicciones a fin de observar las características que deben contener las capacitaciones y evaluaciones correspondientes.

\begin{tabular}{|c|c|c|c|c|c|c|}
\hline 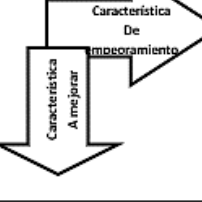 & 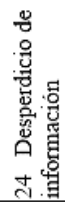 & 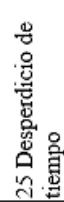 & 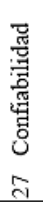 & 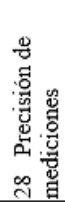 & 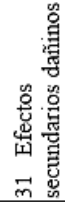 & 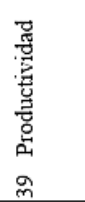 \\
\hline 12 Forma & & $\begin{array}{l}14 \\
10 \\
34 \\
17\end{array}$ & & $\begin{array}{c}28 \\
32 \\
1\end{array}$ & $\begin{array}{c}35, \\
1\end{array}$ & $\begin{array}{l}17,26 \\
34,10\end{array}$ \\
\hline $\begin{array}{l}25 \text { Desperdicio de } \\
\text { tiempo }\end{array}$ & $\begin{array}{l}24 \\
26, \\
28, \\
32\end{array}$ & & & & & \\
\hline 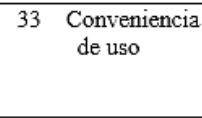 & $\begin{array}{c}4, \\
10 \\
27 \\
22\end{array}$ & $\begin{array}{c}4 \\
28 \\
10 \\
22\end{array}$ & & & & \\
\hline 39 Productividad & & & $\begin{array}{c}1, \\
35, \\
10, \\
38\end{array}$ & & & \\
\hline
\end{tabular}

Tabla 3 Matriz de contradicciones
Ahora bien, una vez aplicada la técnica es posible identificar en la matriz de contradicciones que los principios de inventiva son 14, sin embargo, los que aplican al tema en cuestión, "mejorar la mano de obra" para tener mayor productividad mediante más capacitaciones, son únicamente 9 (1 segmentación, 4 asimetría, 10 acción previa, 14 esferiolidad, 17 moviéndose a una nueva dimensión, 22 convertir algo malo en un beneficio, 24 mediador, 26 copiado y 32 cambio de color), con los cuales se puede asegurar que las capacitaciones sean eficientes tomando de pauta los siguientes criterios:

- Segmentación de las capacitaciones las cuales se deben enfocar en mayor instancia a los talleres para así cumplir con los demás criterios.

- Acción anticipada para que en las capacitaciones sean enfocadas a talleres en su mayoría y no conferencias, pues estas son las que dejan más riqueza intelectual y cumplen su cometido en todas las variables analizadas.

- Que sean asimétricas, tanto las capacitaciones como las evaluaciones 360 , pues en la fase 4 se identificó la importancia de las mediciones posteriores a las capacitaciones y talleres.

- La esferilidad en las capacitaciones, que estas sean a diferentes colaboradores y así cíclicamente hasta que existan especialistas en cada tema o área.

- En los talleres, potenciar los efectos negativos que tiene la falta de talleres tanto en el desarrollo profesional, como laboral, así como los riesgos de no tomarlas.

- Utilizar simuladores en los talleres, pues estos reafirmaran el punto de los riesgos de los efectos negativos además que capta la atención de ambos hemisferios del cerebro absorbiendo mejor el conocimiento impartido. 
- Identificar a los especialistas con alguna insignia de color además de los nombramientos que quedan solo en una constancia, así, el personal nuevo, sabrá a quien acudir ante una contingencia mejorando el sentido de pertenencia con la empresa y la cultura laboral interna y a los nuevos ingresos.

Sexta fase: Aplicar la gestión mediante la metodología de Balanced ScoreCard, el cual tendrá como principales perspectivas el índice de capacitaciones, análisis de puestos, trabajo en equipo, productividad, motivación y cultura, De la misma forma, cada una de estas tendrá un indicador principal con la finalidad de que sirva como indicador para monitorear el cumplimiento de objetivo propuesto en la presente investigación. En este contexto, para la creación del cuadro integral de mando, las ponderaciones para cada perspectiva serán en una escala de 1 al 10, siendo estas como más importante la productividad con 10, las capacitaciones con 10, la cultura con 8 , el análisis de puestos con 7, el trabajo en equipo 7 y la motivación 5 . Un valor objetivo de $95 \%$ y un valor inicial de $80 \%, 70 \%, 50 \%, 90 \%, 50 \%$ y $80 \%$ respectivamente.

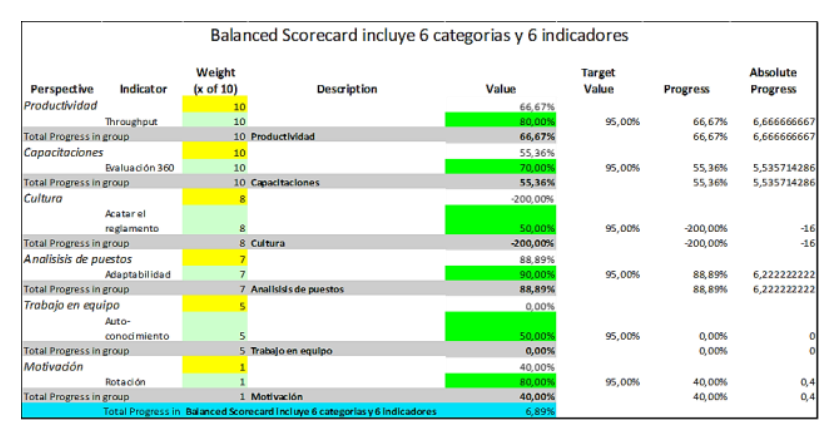

Figura 8 Progreso del Balanced ScoreCrad

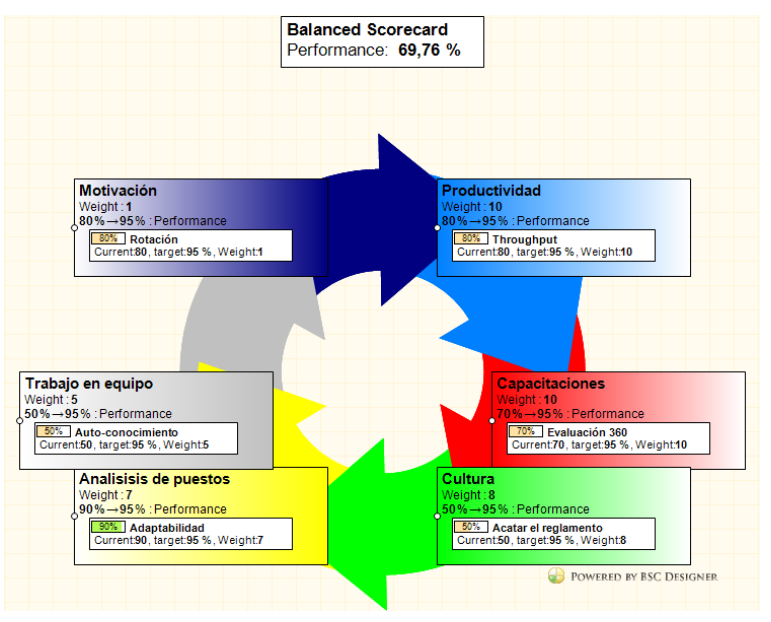

Figura 9 Cuadro integral de mando del rendimiento total
Como interpretación final, al aplicar la metodología es posible identificar que tomando de referencia los valores de peso de la perspectiva, los datos iniciales, base de indicador y su objetivo a cumplir en porcentajes, ya se cuenta con un avance de $6.89 \%$ para alcanzar las metas planeadas.

Por su parte, el cuadro integral de mando, mantiene un rendimiento total del $69.76 \%$, lo que representa un $70 \%$ de cumplimiento de los objetivos para las 6 perspectivas, en este sentido, es importante identificar que, si bien es cierto que es un avance total significativo, también el acatar el reglamento es el indicador cultural al que hay que tener mayor cuidado, pues se encuentra $200 \%$ por debajo acorde a su línea base mínima aceptable y el peso de la perspectiva., por su parte, el auto conocimiento, indicador de la perspectiva trabajo en equipo, no tiene aún ningún avance pues está actualmente en $0 \%$ al no aumentar del 50\% de valor y línea base.

Séptima fase: Análisis del procedimiento científico., en la presente investigación, se ha puesto a prueba la importancia del estudio científico riguroso, el cual comienza con la identificación de las características de calidad que demanda la empresa cuestión, para así detectar que la mano de obra, es lo que está afectando en mayor ponderación, en este sentido, cabe recordar que, como se mencionó al principio, este tipo de investigación pude ser realizada por un sinfín de profesionistas, pues la tecnología que se ha utilizado para poder llevar a cabo un estudio riguroso es la utilización del software QFD Capture para realizar QFD., de la misma forma, para realizar el diagrama de forrester y la simulación, se utilizó VenSim., para el diseño de experimentos se superficie de respuesta y prueba de hipótesis de chi cuadrado, se utilizó Minitab., y para Balanced ScoreCard BSC Designer., siendo únicamente los cálculos manuales de la técnica TRIZ,. Además del tamaño de la muestra para poblaciones finitas, el tipo de muestreo que se utilizó fue simple, por ende, las variables de investigación fueron recaudadas a consideración del investigador, siendo el tamaño de la población, el número de variables con las que cuenta el diagrama de forrester (101), la hipótesis general, $\mathrm{H}_{0}$ y $\mathrm{H}_{1}$ son mostradas en los anexos. 
Sumando aún y a fin de que el lector cuente con más herramientas para el estudio científico, cabe señalar que es posible utilizar en este caso análisis múltiples de varianza, AMEF para servicio, diagramas PERT, kanban, poka-yokes y la ecuación de regresión que proporciona el análisis de diseño de experimentos de la tercera fase para poder predecir el comportamiento de las variables en un estado especifico.

Por lo cual se puede concluir que la efectividad de la presente investigación es del $95 \%$, pues fue el porcentaje que se utilizó en todos los modelos estadísticos, aceptando así la hipótesis alternativa y rechazando la hipótesis nula, dejando, si el lector así lo considera, una forma de investigar sustentada y comprobable de manera eficaz, eficiente $y$, sobre todo, sencilla.

\section{Anexos}

Tamaño de la muestra.

$\mathrm{N}=$ tamaño de la población 101

$\mathrm{n}=$ Tamaño de la muestra

$\mathrm{Z}=$ Valor de significancia $95 \%=1.96$

$\mathrm{e}=$ Error aceptable $5 \%=0.05$

$\sigma=$ Desviacion estandar 0.5

$n=\frac{N \sigma^{2} Z^{2}}{(N-1) e^{2}+\sigma^{2} Z^{2}}$

$n=\frac{(101)(0.5)^{2}(1.96)^{2}}{(101-1) 0.05^{2}+0.5^{2} 1.96^{2}}$

$\mathrm{n}=80.13=80$ datos a analizar

\section{Tipo de muestreo}

El tipo de muestreo que se seleccionara en la presente investigación es de tipo simple, por lo cual, para obtener el total de los datos necesarios proporcionados por el tamaño de la muestra, se seleccionaran uno a uno sucesivamente hasta obtener los 80 datos.

\section{Variables de investigación}

Por su parte, las variables de investigación están segmentadas en 8 categorías significativas, por lo cual se realizará la proporcionalidad para cada una de ellas de la siguiente forma. $p=\frac{\text { total de valores }}{\text { total de categorias }}$

$p=\frac{80}{8}$

$\mathrm{P}=10$ datos por categoría.

1. Capacitación.

2. Análisis de puestos.

3. Trabajo en equipo.

4. Productividad.

5. Costos.

6. Motivación.

7. Cultura.

8. Jornada laboral.

\begin{tabular}{|c|c|c|c|c|c|}
\hline \multicolumn{6}{|c|}{ Capacitaciones } \\
\hline Criterios & \multicolumn{2}{|c|}{2017} & \multicolumn{2}{|l|}{2018} & \multirow{2}{*}{$\begin{array}{l}2019 \\
\text { ler } \\
\text { semest } \\
\text { re }\end{array}$} \\
\hline Capacitación & $\begin{array}{l}\text { 1er } \\
\text { semest } \\
\text { re }\end{array}$ & $\begin{array}{l}\text { 2do } \\
\text { semestr } \\
\text { e }\end{array}$ & $\begin{array}{l}\text { ler } \\
\text { semest } \\
\text { re }\end{array}$ & $\begin{array}{l}2 \text { do } \\
\text { semestr } \\
\text { e }\end{array}$ & \\
\hline Interna & 1 & 1 & 2 & 1 & 1 \\
\hline Externa & 1 & 0 & 0 & 1 & 0 \\
\hline Inducción & 0 & 1 & 0 & 0 & 0 \\
\hline Gubernamentales & 1 & 0 & 1 & 0 & 1 \\
\hline Períodicas & 0 & 0 & 1 & 0 & 0 \\
\hline \multicolumn{6}{|l|}{ Evaluación } \\
\hline Diagnostico & 0 & 1 & 0 & 0 & 0 \\
\hline Formativa & 1 & 1 & 0 & 2 & 1 \\
\hline Sumativa & 1 & 0 & 2 & 0 & 1 \\
\hline Continua & 0 & 0 & 1 & 0 & 2 \\
\hline Post-Capacitación & 3 & 2 & 4 & 2 & 2 \\
\hline \multicolumn{6}{|l|}{ Análisis de puestos } \\
\hline Criterios & \multicolumn{2}{|l|}{2017} & \multicolumn{2}{|l|}{2018} & 2019 \\
\hline Asignación de tareas & $\begin{array}{l}\text { ler } \\
\text { semest } \\
\text { re }\end{array}$ & $\begin{array}{l}2 \mathrm{do} \\
\text { semestr } \\
\mathrm{e}\end{array}$ & $\begin{array}{l}\text { ler } \\
\text { semest } \\
\text { re }\end{array}$ & $\begin{array}{l}2 \mathrm{do} \\
\text { semestr } \\
\text { e }\end{array}$ & $\begin{array}{l}\text { ler } \\
\text { semest } \\
\text { re }\end{array}$ \\
\hline Desenvovimiento & 6 & 6 & 6 & 8 & 8 \\
\hline Habilidades & 6 & 7 & 7 & 7 & 7 \\
\hline Destrezas & 7 & 7 & 8 & 8 & 8 \\
\hline Capacides & 5 & 6 & 6 & 7 & 7 \\
\hline Ergonomía & 1 & 1 & 1 & 1 & 1 \\
\hline \multicolumn{6}{|l|}{$\begin{array}{lll}\begin{array}{l}\text { Relación } \\
\text { puestos }\end{array} & \text { con } & \text { otros } \\
\end{array}$} \\
\hline Comunicación & 6 & 7 & 7 & 7 & 8 \\
\hline Ordenación de personal & 5 & 5 & 6 & 6 & 6 \\
\hline Descripción de puestos & 2 & 3 & 3 & 4 & 4 \\
\hline Conocimiento & 5 & 5 & 6 & 6 & 6 \\
\hline Experiencia & $60 \%$ & $70 \%$ & $80 \%$ & $80 \%$ & $80 \%$ \\
\hline \multicolumn{6}{|l|}{ Trabajo en equipo } \\
\hline Criterios & \multicolumn{2}{|l|}{2017} & \multicolumn{2}{|l|}{2018} & 2019 \\
\hline Liderazgo & $\begin{array}{l}\text { ler } \\
\text { semest } \\
\text { re }\end{array}$ & $\begin{array}{l}\text { 2do } \\
\text { semestr } \\
\text { e }\end{array}$ & $\begin{array}{l}\text { ler } \\
\text { semest } \\
\text { re }\end{array}$ & $\begin{array}{l}\text { 2do } \\
\text { semestr } \\
\text { e }\end{array}$ & $\begin{array}{l}\text { ler } \\
\text { semest } \\
\text { re }\end{array}$ \\
\hline Tipos de liderazgo & $2 \%$ & $3 \%$ & $3 \%$ & $4 \%$ & $5 \%$ \\
\hline $\begin{array}{lll}\begin{array}{l}\text { Influencia } \\
\text { demás }\end{array} & \text { sobre los } \\
\end{array}$ & $6 \%$ & $4 \%$ & $3 \%$ & $3 \%$ & $2 \%$ \\
\hline Solución de problemas & $8 \%$ & $6 \%$ & $6 \%$ & $5 \%$ & $5 \%$ \\
\hline Conocimiento & $2 \%$ & $4 \%$ & $6 \%$ & $7 \%$ & $8 \%$ \\
\hline Auto-conocimiento & $2 \%$ & $4 \%$ & $4 \%$ & $5 \%$ & $6 \%$ \\
\hline \multicolumn{6}{|l|}{ Clima organizacional } \\
\hline Condiciones de trabajo & $1 \%$ & $2 \%$ & $3 \%$ & $3 \%$ & $5 \%$ \\
\hline Compromiso & $6 \%$ & $4 \%$ & $3 \%$ & $3 \%$ & $2 \%$ \\
\hline Sentido de pertenencia & $7 \%$ & $4 \%$ & $4 \%$ & $3 \%$ & $2 \%$ \\
\hline Orientación al servicio & $0 \%$ & $0 \%$ & $1 \%$ & $1 \%$ & $2 \%$ \\
\hline Orientación al resultado & $3 \%$ & $3 \%$ & $4 \%$ & $5 \%$ & $6 \%$ \\
\hline \multicolumn{6}{|l|}{ Productividad } \\
\hline Criterios & \multicolumn{2}{|l|}{2017} & \multicolumn{2}{|l|}{2018} & 2019 \\
\hline Capital de trabajo & $\begin{array}{l}\text { ler } \\
\text { semest } \\
\text { re }\end{array}$ & $\begin{array}{l}\text { 2do } \\
\text { semestr } \\
\text { e }\end{array}$ & $\begin{array}{l}\text { ler } \\
\text { semest } \\
\text { re }\end{array}$ & $\begin{array}{l}\text { 2do } \\
\text { semestr } \\
\text { e }\end{array}$ & $\begin{array}{l}\text { ler } \\
\text { semest } \\
\text { re }\end{array}$ \\
\hline
\end{tabular}

GUTIÉRREZ-GARCÍA, Alfredo. La gestión de la calidad mediante métodos científicos. Revista de Educación Técnica. 2019 


\begin{tabular}{|c|c|c|c|c|c|}
\hline Infraestructura & $60 \%$ & $63 \%$ & $65 \%$ & $67 \%$ & $70 \%$ \\
\hline $\begin{array}{ll}\begin{array}{l}\text { Administración } \\
\text { recursos }\end{array} & \text { de } \\
\end{array}$ & $10 \%$ & $10 \%$ & $15 \%$ & $20 \%$ & $30 \%$ \\
\hline Solvencia & $5 \%$ & $5 \%$ & $10 \%$ & $15 \%$ & $20 \%$ \\
\hline Inventarios & $10 \%$ & $10 \%$ & $5 \%$ & $5 \%$ & $15 \%$ \\
\hline $\begin{array}{l}\text { Apoyos } \\
\text { gubernamentales }\end{array}$ & $0 \%$ & $0 \%$ & $0 \%$ & $0 \%$ & $0 \%$ \\
\hline $\begin{array}{ll}\text { Tiempos } & \mathbf{y} \\
\text { movimientos } & \end{array}$ & & & & & \\
\hline Eficientes & $5 \%$ & $5 \%$ & $10 \%$ & $10 \%$ & $8 \%$ \\
\hline Ineficientes & $-2 \%$ & $-5 \%$ & $-2 \%$ & $-4 \%$ & $-5 \%$ \\
\hline Proactividad & $2 \%$ & $2 \%$ & $5 \%$ & $5 \%$ & $9 \%$ \\
\hline Asignación de tareas & $2 \%$ & $2 \%$ & $5 \%$ & $5 \%$ & $5 \%$ \\
\hline Piezas elaboradas & $10 \%$ & $5 \%$ & $15 \%$ & $5 \%$ & $5 \%$ \\
\hline \multicolumn{6}{|l|}{ Costos } \\
\hline Criterios & \multicolumn{2}{|l|}{2017} & \multicolumn{2}{|l|}{2018} & 2019 \\
\hline Activos ajenos & $\begin{array}{l}\text { ler } \\
\text { semest } \\
\text { re }\end{array}$ & $\begin{array}{l}\text { do } \\
\text { semestr } \\
\text { e }\end{array}$ & $\begin{array}{l}\text { ler } \\
\text { semest } \\
\text { re }\end{array}$ & $\begin{array}{l}\text { do } \\
\text { semestr } \\
\text { e }\end{array}$ & $\begin{array}{l}\text { ler } \\
\text { semest } \\
\text { re }\end{array}$ \\
\hline Outsourcing & $10 \%$ & $20 \%$ & $20 \%$ & $20 \%$ & $40 \%$ \\
\hline Capacitación externa & $10 \%$ & $0 \%$ & $10 \%$ & $0 \%$ & $10 \%$ \\
\hline Free lance & $10 \%$ & $0 \%$ & $0 \%$ & $0 \%$ & $10 \%$ \\
\hline Maquilar & $0 \%$ & $0 \%$ & $0 \%$ & $0 \%$ & $0 \%$ \\
\hline Apoyo en mejora de MP & $40 \%$ & $40 \%$ & $50 \%$ & $70 \%$ & $80 \%$ \\
\hline \multicolumn{6}{|l|}{ Gastos de operación } \\
\hline Logística & $20 \%$ & $20 \%$ & $30 \%$ & & \\
\hline RRHH & $5 \%$ & $5 \%$ & $5 \%$ & $6 \%$ & $6 \%$ \\
\hline Maquinaría y equipo & $5 \%$ & $6 \%$ & $6 \%$ & $7 \%$ & $7 \%$ \\
\hline Mantenimiento & $4 \%$ & $5 \%$ & $5 \%$ & $6 \%$ & $6 \%$ \\
\hline Almácen & $5 \%$ & $6 \%$ & $6 \%$ & $7 \%$ & $8 \%$ \\
\hline \multicolumn{6}{|l|}{ Motivación } \\
\hline Criterios & \multicolumn{2}{|l|}{2017} & \multicolumn{2}{|l|}{2018} & 2019 \\
\hline Incentivos & $\begin{array}{l}\text { ler } \\
\text { semest } \\
\text { re }\end{array}$ & $\begin{array}{l}\text { do } \\
\text { semestr } \\
\text { e }\end{array}$ & $\begin{array}{l}\text { ler } \\
\text { semest } \\
\text { re }\end{array}$ & $\begin{array}{l}\text { 2do } \\
\text { semestr } \\
\text { e }\end{array}$ & $\begin{array}{l}\text { ler } \\
\text { semest } \\
\text { re }\end{array}$ \\
\hline Economía de la empresa & $0 \%$ & $1 \%$ & $1 \%$ & $2 \%$ & $3 \%$ \\
\hline $\begin{array}{ll}\begin{array}{l}\text { Seguimiento } \\
\text { empleados }\end{array} & \text { de } \\
\end{array}$ & $0 \%$ & $1 \%$ & $1 \%$ & $4 \%$ & $5 \%$ \\
\hline Mayor eficiencia laboral & $3 \%$ & $5 \%$ & $6 \%$ & $6 \%$ & $7 \%$ \\
\hline Políticas internas & $6 \%$ & $4 \%$ & $3 \%$ & $2 \%$ & $2 \%$ \\
\hline $\begin{array}{l}\text { Incremento de la } \\
\text { demanda en empleados }\end{array}$ & $1 \%$ & $1 \%$ & $2 \%$ & $3 \%$ & $3 \%$ \\
\hline \multicolumn{6}{|l|}{ Horarios fijos } \\
\hline $\begin{array}{l}\text { Ejecución de las tareas } \\
\text { asignadas }\end{array}$ & $4 \%$ & $4 \%$ & $5 \%$ & $6 \%$ & $7 \%$ \\
\hline $\begin{array}{lll}\text { No retrasos } & \text { en } \\
\text { producción }\end{array}$ & $3 \%$ & $3 \%$ & $4 \%$ & $4 \%$ & $5 \%$ \\
\hline $\begin{array}{l}\text { Evitar pago de horas } \\
\text { extras }\end{array}$ & $0 \%$ & $0 \%$ & $1 \%$ & $1 \%$ & $2 \%$ \\
\hline Reducción de estrés & $0 \%$ & $0 \%$ & $0 \%$ & $1 \%$ & $2 \%$ \\
\hline Reducción de rotación & $3 \%$ & $4 \%$ & $4 \%$ & $5 \%$ & $7 \%$ \\
\hline \multicolumn{6}{|l|}{ Cultura } \\
\hline Criterios & \multicolumn{2}{|l|}{2017} & \multicolumn{2}{|l|}{2018} & 2019 \\
\hline Higiene & $\begin{array}{l}\text { ler } \\
\text { semest } \\
\text { re }\end{array}$ & $\begin{array}{l}\text { 2do } \\
\text { semestr } \\
\text { e }\end{array}$ & $\begin{array}{l}\text { 1er } \\
\text { semest } \\
\text { re }\end{array}$ & $\begin{array}{l}\text { 2do } \\
\text { semestr } \\
\text { e }\end{array}$ & $\begin{array}{l}\text { 1er } \\
\text { semest } \\
\text { re }\end{array}$ \\
\hline $\begin{array}{ll}\begin{array}{l}\text { Acatar reglas } \\
\text { indicaciones }\end{array} & \text { e } \\
\end{array}$ & $4 \%$ & $4 \%$ & $5 \%$ & $5 \%$ & $5 \%$ \\
\hline Mantenimiento & $8 \%$ & $8 \%$ & $9 \%$ & $9 \%$ & $9 \%$ \\
\hline $\begin{array}{ll}\begin{array}{l}\text { Desinfección } \\
\text { materiales }\end{array} & \text { de } \\
\end{array}$ & $8 \%$ & $9 \%$ & $9 \%$ & $9 \%$ & $9 \%$ \\
\hline Hábitos personales & $2 \%$ & $2 \%$ & $3 \%$ & $3 \%$ & $3 \%$ \\
\hline $\begin{array}{l}\text { Organización de área de } \\
\text { trabajo }\end{array}$ & $7 \%$ & $7 \%$ & $8 \%$ & $8 \%$ & $8 \%$ \\
\hline \multicolumn{6}{|l|}{$\begin{array}{ll}\begin{array}{l}\text { Superación } \\
\text { personal }\end{array} & \text { de }\end{array}$} \\
\hline Escolaridad & $6 \%$ & $7 \%$ & $7 \%$ & $7 \%$ & $7 \%$ \\
\hline Puntualidad & $7 \%$ & $7 \%$ & $8 \%$ & $8 \%$ & $8 \%$ \\
\hline Estatus social & $3 \%$ & $3 \%$ & $3 \%$ & $3 \%$ & $3 \%$ \\
\hline Actitudes & $3 \%$ & $3 \%$ & $3 \%$ & $3 \%$ & $3 \%$ \\
\hline Valores & $4 \%$ & $5 \%$ & $5 \%$ & $5 \%$ & $5 \%$ \\
\hline \multicolumn{6}{|l|}{ Jornadas laborales } \\
\hline Criterios & 2017 & & 2018 & & 2019 \\
\hline Prestación de ley & $\begin{array}{l}\text { ler } \\
\text { semest } \\
\text { re }\end{array}$ & $\begin{array}{l}\text { 2do } \\
\text { semestr } \\
\text { e }\end{array}$ & $\begin{array}{l}\text { ler } \\
\text { semest } \\
\text { re }\end{array}$ & $\begin{array}{l}\text { 2do } \\
\text { semestr } \\
\text { e }\end{array}$ & $\begin{array}{l}\text { ler } \\
\text { semest } \\
\text { re }\end{array}$ \\
\hline Fijación de horarios & $20 \%$ & $20 \%$ & $-10 \%$ & $10 \%$ & $10 \%$ \\
\hline Seguro médico & $80 \%$ & $80 \%$ & $80 \%$ & $80 \%$ & $80 \%$ \\
\hline Pago de nómina & $90 \%$ & $90 \%$ & $90 \%$ & $90 \%$ & $90 \%$ \\
\hline Vacaciones & $80 \%$ & $80 \%$ & $90 \%$ & $90 \%$ & $90 \%$ \\
\hline Contrato laboral & $90 \%$ & $90 \%$ & $90 \%$ & $90 \%$ & $100 \%$ \\
\hline $\begin{array}{l}\text { Organización } \\
\text { procesos }\end{array}$ & & & & & \\
\hline Fijar organigrama & $20 \%$ & $20 \%$ & $20 \%$ & $20 \%$ & $20 \%$ \\
\hline Organizar tiempos & $30 \%$ & $30 \%$ & $30 \%$ & $40 \%$ & $40 \%$ \\
\hline $\begin{array}{l}\text { Estableces director de } \\
\text { actividad }\end{array}$ & $20 \%$ & $20 \%$ & $30 \%$ & $30 \%$ & $30 \%$ \\
\hline Hacer PDCA & $50 \%$ & $-10 \%$ & $40 \%$ & $50 \%$ & $60 \%$ \\
\hline Plan de actividades & $60 \%$ & $60 \%$ & $60 \%$ & $70 \%$ & $70 \%$ \\
\hline
\end{tabular}

Tabla 4 Datos recolectados para estudio

\section{Hipótesis general}

¿La diversidad de técnicas de innovación utilizadas en la investigación promoverán una innovación al sistema de producción de la empresa Quesos el Ángel con un nivel de significancia del 95\%?

\section{Hipótesis nula}

Las técnicas de innovación implementadas en la investigación no promueven una mejora en el sistema de producción de la empresa Quesos el Ángel con un nivel de significancia del 95\%

\section{Hipótesis alternativa}

Al menos una de las técnicas de innovación implementadas en la presente investigación promueve una mejora en el sistema de producción de la empresa Quesos el Ángel con un nivel de significancia del $95 \%$

\section{Referencias}

Antonio, F. (2010). El Balanced Scorecard como herramienta del siglo XXI. Gestión y Desarrollo. Recuperado el 18 de Julio de 2019

García, J. M. (2003). Teoría y ejercicios prácticos de dinámica de sistemas. Recuperado el 18 de Julio de 2019

Gutiérrez, H. (s.f.). Análisis y diseño de experimentos (2 ed.). México: McGraw Hill. Recuperado el 18 de Julio de 2019

Huerta, J. D. (2015). QFD Desplegue de la función de la calidad. Chile: JD Salinas. Recuperado el 18 de Julio de 2019

Oropeza, R. (2011). NIÑOS Y JÓVENES CREATIVOS E INNOVADORES EN UN TRIS, CON TRIZ. México. Recuperado el 18 de Julio de 2019 